\title{
Central bronchiectasis in allergic bronchopulmonary aspergillosis: comparative evaluation of computed tomography of the thorax with bronchography
}

\author{
N. Panchal*, C. Pant**, R. Bhagat*, A. Shah*
}

Central bronchiectasis in allergic bronchopulmonary aspergillosis: comparative evaluation of computed tomography of the thorax with bronchography. N. Panchal, C. Pant, R. Bhagat, A. Shah. CERS Journals Ltd 1994.

ABSTRACT: Demonstration of central bronchiectasis (CB) with normal peripheral bronchi is an essential requirement for the diagnosis of allergic bronchopulmonary aspergillosis (ABPA). Although the results of bronchography remain the gold standard for demonstration of central bronchiectasis they are not always diagnostic. Moreover, it is an unpleasant invasive procedure which may be difficult to perform in a patient of allergic bronchopulmonary aspergillosis with acute severe asthma. In an attempt to find a safe and effective alternative to demonstrate central bronchiectasis computed tomography (CT) of the thorax was evaluated against bronchography.

Twenty one patients with allergic bronchopulmonary aspergillosis underwent computed tomography of the thorax followed by bronchography. Of the 378 bronchopulmonary segments available for analysis, 42 had to be excluded because of consolidation or non-filling of the contrast dye, leaving 336 segments for evaluation.

CB was identified on CT in all 21 patients. Detailed analysis of the visualized segments revealed that computed tomography (using $8 \mathrm{~mm}$ contiguous scans) identified 146 of the 212 segments showing central bronchiectasis on bronchography (sensitivity 70\%) and 114 of the 124, read as normal on bronchography (specificity $92 \%$ ). Supplemental $4 \mathrm{~mm}$ scans, used in 8 out of 21 patients improved the overall sensitivity of computed tomography to $83 \%$, whilst the specificity remained unchanged at $92 \%$.

Thus, computed tomography of the thorax, being more acceptable to the patient, has the potential of being the investigation of choice for the demonstration of central bronchiectasis in patients with allergic bronchopulmonary aspergillosis.

Eur Respir J., 1994, 7, 1290-1293.

Central bronchiectasis $(\mathrm{CB})$ with normal peripheral bronchi, a pathognomic feature of allergic bronchopulmonary aspergillosis (ABPA), which characteristically involves the medium-sized bronchi of 3rd and 4th generation, was first described by ScADDING [1]. In fact, demonstration of $\mathrm{CB}$, is an essential requirement for the diagnosis of ABPA [2]. Bronchography had been considered the gold standard for the demonstration of $\mathrm{CB}$, until recently [3]. However, it is an unpleasant, invasive procedure, requiring special skills and can have potentially fatal adverse effects [4]. Furthermore, this procedure may be difficult to perform in a patient of ABPA presenting with acute severe asthma [5].

Computed tomography (CT) has now emerged as an effective alternative in the diagnosis of bronchiectasis [6-13]. However, no comparative study has been reported, so far, on patients with ABPA. This study was, therefore, carried out to evaluate the role of $\mathrm{CT}$ of the thorax in demonstration of CB in ABPA, and the results were compared with those of bronchography, the gold standard.

\section{Patients}

*Dept of Clinical Research, Vallabhbhai Patel Chest Institute, University of Delhi, Delhi, India. **Imaging Division, Institute of Nuclear Medicine and Allied Sciences, Timarpur, Delhi, India.

Correspondence: N. Panchal

B-80, Swasthya Vihar

Vikas Marg

Transyamuna

Delhi (East) - 110092

India

Keywords: Allergic bronchopulmonary aspergillosis

bronchography

central bronchiectasis

computed tomography (CT) of the thorax

Received: November 111993

Accepted after revision March 281994

The study comprised 21 consecutive patients with ABPA. The patients were considered to have ABPA, in view of the following criteria: 1) a history of asthma; 2) peripheral blood eosinophilia; 3) transient pulmonary infiltrates; 4) presence of Type I cutaneous reactivity to aspergillin, with or without Type III reaction; 5) demonstration of serum precipitins against Aspergillus spp.; and 6) elevated serum immunoglobulin E (IgE) levels. Patients with a history of smoking, associated systemic disease and pregnancy were excluded. Consent was obtained from each patient and the study was approved by the Research Cell of the Institute.

There were 14 male and 7 female patients, with a mean age of 36 yrs (range 13-55 yrs). The mean duration of illness was 13 yrs (range $1-45$ yrs). All of the 21 patients had history of asthma, transient pulmonary infiltrates, 
type I skin reaction to aspergillin and elevated total serum IgE levels. Peripheral blood eosinophilia was present in 18 out of 21 patients, and serum precipitins against Aspergillus spp. were demonstrable in 16 out of 21 patients. Type III skin reactivity was observed in 18 out of 21 patients. The 21 patients were clinically staged as follows: Stage I $(n=1)$; Stage II $(n=7)$; Stage III $(n=12)$; Stage IV $(n=0)$; and Stage V $(n=1)$.

\section{Computed tomography}

Non-contrast computed tomography of the thorax, was performed on a Somatom-DRH CT scanner (Siemens, Erlangen, Germany), with a 512 matrix size. CT scans

a)

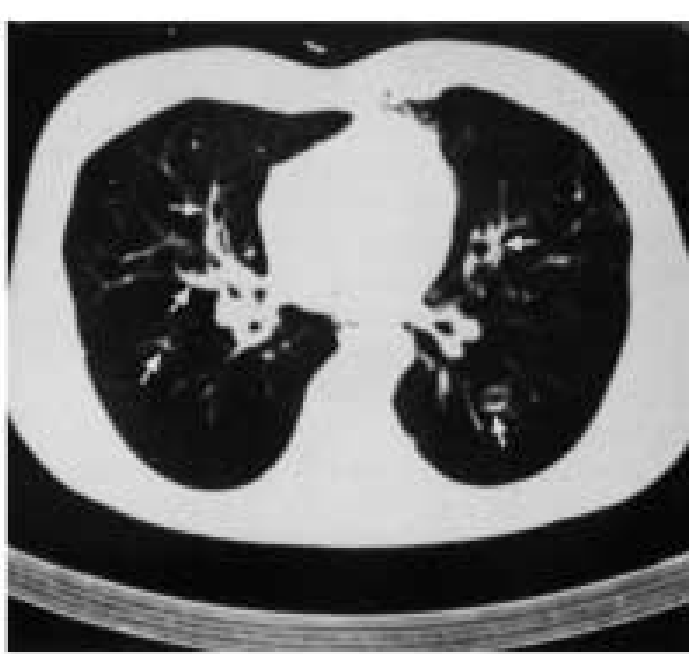

b)

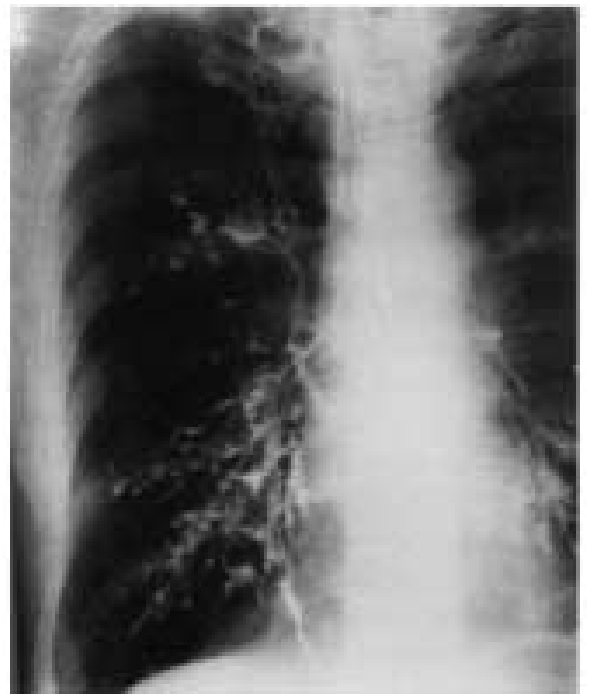

Fig. 1. - a) Chest computed tomographic (CT) scan obtained at the level of the right middle lobe bronchus, showing central bronchiectasis (arrows) involving the medial and lateral segmental bronchi of the right middle lobe, right lateral basal segmental bronchus, left superior lingular segmental bronchus. Note the normal lung periphery on both sides. b) Right-sided bronchogram of the same patient, showing central bronchiectasis with normal peripheral tapering in all the segmental bronchi, of all three lobes. were obtained at $125 \mathrm{kV}, 210 \mathrm{~mA}$, with a scan time of 3-5 s, in supine position at full end-inspiration, from lung apices to the bases, with $8 \mathrm{~mm}$ contiguous sections. In eight cases, areas of interest were scanned with 4 $\mathrm{mm}$ contiguous slices. Individual bronchopulmonary segments were identified by the anatomical division of the appropriate lobar bronchus and its relationship to the major and minor fissures [14]. Bronchiectasis was identified, as described by NAIDICH et al. [6]: 1) signet ring appearance; 2) string of pearl appearance; and 3) beaded/tubular opacities (figs. 1a and 2). CB with normal peripheral bronchi, was reported after examining contiguous serial scans and ascertaining the distance of the lesions from the hilum.

\section{Bronchography}

Bronchography was performed within 2 weeks of the CT procedure, after stabilizing the patient with steroids and bronchodilators. It was performed via a nasotracheal catheter, after premedication with atropine $(0.01$ $\mathrm{mg} \cdot \mathrm{kg}^{-1}$ ), using Dionosil oily (propyliodone, Glaxo Laboratories, UK). Intravenous hydrocortisone and theophylline were given prior to the procedure. Bilateral bronchograms were obtained in all of the 21 patients. Anteroposterior, lateral and oblique views were taken for both sides. Bronchograms were interpreted at segmental level for the presence or absence of central bronchiectasis with normal peripheral filling of the dye (fig 1b).

Analysis

All of the CT scans and bronchograms were interpreted for the presence/absence of $\mathrm{CB}$, by a team comprising

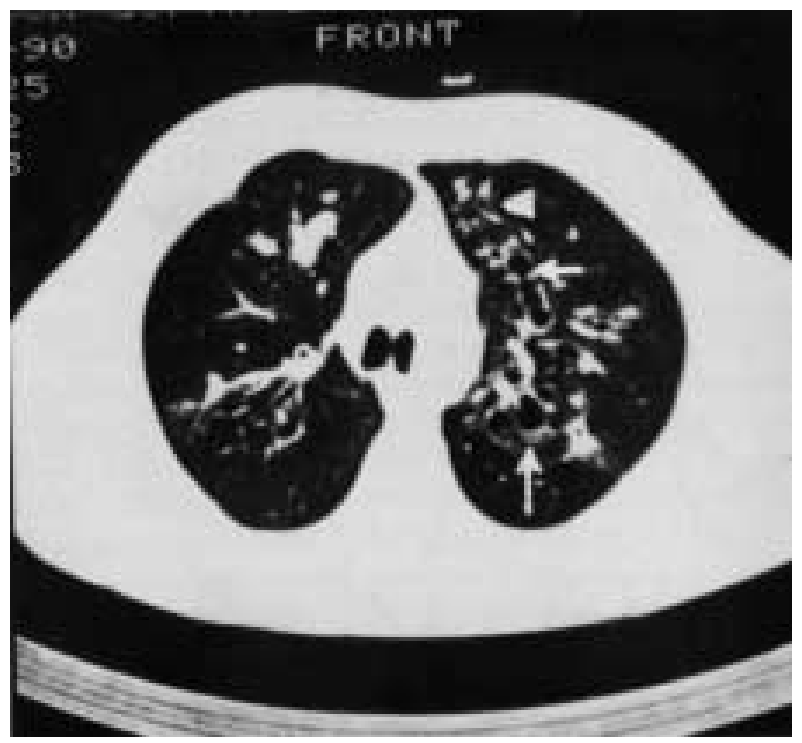

Fig. 2. - Chest computed tomographic (CT) scan of another patient, at the level of carina, showing central bronchiectasis involving the anterior segmental bronchus (short arrow) and apicoposterior segmental bronchus (long arrow) of the left upper lobe. Note the normal lung periphery (arrow head). 
both radiologists and pneumologists. The CT scans were read first in a random order, followed by the bronchograms, which were also read in a random fashion. The reading was done on the basis of consensus opinion, and, where there was disagreement, the segment was taken as nonbronchiectatic. Bronchography was regarded as the gold standard, against which CT was judged. The sensitivity and specificity of CT were determined at segmental level.

\section{Results}

CB was visualized by CT in all 21 patients included in the study. For further analysis at the segmental level, a total of 378 segments were available for study. Since bronchography was regarded as the gold standard for the study, 42 segmental bronchi had to be excluded, as they were not visualized on bronchograms, but only on CT, leaving 336 segmental bronchi for analysis. Bronchography demonstrated CB in 212 segments, whilst the remaining 124 segments were read as normal. Of the 212 segments showing CB on bronchography, 146 segments were reported as having $\mathrm{CB}$ on $\mathrm{CT}$ scans. Sensitivity of CT for detection of $\mathrm{CB}$ was found to be $70 \%$, with a false negative rate of $30 \%$. CT was able to correctly identify 114 of the 124 segments reported as normal by bronchography. Thus, CT, as compared to bronchography, had $92 \%$ specificity, with a false positive rate of $8 \%$. Examination of the CT scans of the 42 segmental bronchi, which were not visualized on bronchography and were thus excluded from analysis, revealed that 20 segmental bronchi had CB whilst 16 were normal. No comments were possible in six segmental bronchi due to consolidation.

With $8 \mathrm{~mm}$ contiguous slices, it required on average 21 scans per patient to visualize the entire thorax. However, in 8 out of 21 patients, $4 \mathrm{~mm}$ scans were also done in selected areas (average 8 scans per patient). Of the 144 segmental bronchi scanned twice, 96 were clearly visualized even with $8 \mathrm{~mm}$ sections. Thus, a $4 \mathrm{~mm}$ collimation of the X-ray beam was helpful in further assessment of 48 segmental bronchi (i.e. $14 \%$ of the total), which were not clearly visualized on the $8 \mathrm{~mm}$ initial sections. A review of the bronchograms of these 48 segmental bronchi, revealed $\mathrm{CB}$ in 36 and the remaining 12 were read as normal. The $4 \mathrm{~mm}$ CT sections were able to correctly identify 30 out of the 36 segmental bronchi with $\mathrm{CB}$, thus altering the overall sensitivity of CT scan from $70 \%$ (146 out of 212) to $83 \%$ (176 out of 212). There was no change in the specificity of CT (92\%), as the $4 \mathrm{~mm} \mathrm{CT}$ scans correctly identified all 12 segmental bronchi as normal.

CT of the thorax, on average, required $10 \mathrm{~min}$ per patient to complete the procedure. It was more acceptable to the patient, as it did not entail any discomfort, nor did it require prior preparation. In contrast, bronchography was a time-consuming invasive procedure, requiring $60 \mathrm{~min}$ for the procedure followed by an observation period of $2 \mathrm{~h}$. In spite of prior preparation and stabilization, almost all patients had distressing cough followed by throat discomfort for $6-8 \mathrm{~h}$. One patient, on one occasion, had a bronchospasm following local anaesthetic spray, for which the bronchography had to be postponed.

\section{Discussion}

Rapid and safe documentation of $\mathrm{CB}$ is crucial to confirm the diagnosis of ABPA. This enables the physician to intervene effectively with aggressive corticosteroid therapy, in order to prevent further lung damage. Bronchography, the accepted gold standard for demonstration of $\mathrm{CB}$ is an invasive and potentially risky procedure in patients with ABPA, as almost all are asthmatics [3].

CB was identified on CT of the thorax in all of our 21 patients, which enabled us to rapidly confirm the diagnosis of ABPA. Using $8 \mathrm{~mm}$ contiguous scans, a sensitivity of $70 \%$ and specificity of $92 \%$ was found. Limited use of $4 \mathrm{~mm}$ scans, in selected areas from 8 patients, increased sensitivity to $83 \%$. For 42 segmental bronchi not visualized by bronchography, either due to non-filling of contrast dye or consolidation of the affected segment, analysis by CT showed 20 with $\mathrm{CB}$ and 16 normal; no comments were possible in 6 segments, due to consolidation.

Although a few studies $[3,15]$ have been made of CT of the thorax in ABPA, none of them was specifically designed to evaluate the role of $\mathrm{CT}$ in demonstration of $\mathrm{CB}$ in comparison to bronchography. In our earlier report [5], CT of the thorax in two children with ABPA during acute severe asthma was compared with bronchograms which were obtained subsequent to recovery. A comparative segmental analysis revealed that CT was able to identify 24 of the 27 segments which showed CB on bronchography. Thus, CT of the thorax enabled an immediate confirmation of the diagnosis in these children.

In recent years, CT of the thorax has emerged as a safe and effective alternative to bronchography for demonstration of bronchiectasis. The sensitivity rate in these studies has ranged from $60-100 \%$, while specificity has been of the order of 90-100\% [7-13]. However, there has not yet been a systematic study to evaluate CT of the thorax in comparison to bronchography for demonstration of $\mathrm{CB}$ in ABPA. In studies of $\mathrm{CT}$ of thorax with the standard $10 \mathrm{~mm}$ sections at $10 \mathrm{~mm}$ intervals for demonstration of bronchiectasis, it was felt that the bronchi were not sharply visualized, due to partial volume averaging effect $[7,8,11,13]$. Furthermore, a gap of $10 \mathrm{~mm}$ between the sections may lead to small bronchiectatic lesions being missed $[8,9,11]$, especially in bronchi that course horizontally as their axes lie parallel to the transverse plane. On the other hand, very thin to medium sections of $2-5 \mathrm{~mm}$ increase the radiation dose to the patient [12]. Our protocol of $8 \mathrm{~mm}$ contiguous sections, with $4 \mathrm{~mm}$ contiguous scans in areas of interest in eight cases, enabled us to visualize the bronchi continuously and clearly with minimal radiation exposure. 
CT of the thorax was found to be more acceptable to the patients in comparison to bronchography, as it is a noninvasive procedure, less time-consuming and requiring no follow-up. Thus, it has the potential of being the investigation of choice in the demonstration of $\mathrm{CB}$ in patients with ABPA.

Acknowledgements: The authors are thankful to H.S. Randhawa and Z.U. Khan, Dept of Medical Mycology, V.P. Chest Institute, for conducting the precipitin studies and to B.D. Sharma for secretarial assistance.

\section{References}

1. Scadding JG. The bronchi in allergic aspergillosis. Scand J Respir Dis 1967; 48: 372-377.

2. Greenberger PA, Patterson R. Allergic bronchopulmonary aspergillosis: model of bronchopulmonary disease with defined serologic, radiologic, pathologic and clinical findings from asthma to fatal destructive lung disease. Chest 1987; 91 (Suppl.): 165S-171S.

3. Neeld DA, Goodman LR, Gurney JW, Greenberger PA, Fink JN. Computerized tomography in the evaluation of allergic bronchopulmonary aspergillosis. Am Rev Respir Dis 1990; 142: 1200-1205.

4. Ansell G. Bronchography. In: Ansell G, ed. Complications in Diagnostic Radiology. Oxford, Blackwell Scientific Publications, 1976; pp. 278-300.

5. Shah A, Pant CS, Bhagat R, Panchal N. CT in childhood allergic bronchopulmonary aspergillosis. Pediatr Radiol 1992; 22: 227-228
6. Naidich DP, Zerhauni EA, Siegelman SS, (eds). In: Computed Tomography of the Thorax. 1st edn. New York, Raven Press, 1984; pp. 103-107.

7. Muller NL, Bergin CJ, Ostrow DN, Nichols DM. Role of computed tomography in the recognition of bronchiectasis. AJR 1984; 143: 971-976.

8. Mootoosamy IM, Reznek RH, Osman J, Rees RSO, Green M. Assessment of bronchiectasis by computed tomography. Thorax 1985; 40: 920-924.

9. Grenier P, Maurice F, Musset D, Menu Y, Nahum H. Bronchiectasis: assessment by thin section CT. Radiology 1986; 161: 95-99.

10. Phillips MS, Williams MP, Flower CDR. How useful is computed tomography in the diagnosis and assessment of bronchiectasis. Clin Radiol 1986; 37: 321325.

11. Cooke JC, Currie DC, Morgan AD, et al. Role of computed tomography in diagnosis of bronchiectasis. Thorax 1987; 42: 272-277.

12. Joharjy IA, Bashi SA, Abdullah AK. Value of medium-thickness $\mathrm{CT}$ in the diagnosis of bronchiectasis. AJR 1987; 149: 1133-1137.

13. Munro NC, Cooke JC, Currie DC, Strickland B, Cole PJ. Comparison of thin section computed tomography with bronchography for identifying bronchiectatic segments in patients with chronic sputum production. Thorax 1990; 45: 135-139.

14. Osborne D, Vock P, Godwin JD, Silverman PM. CT identification of bronchopulmonary segments in 50 normal subjects. AJR 1984; 142: 47-52.

15. Currie DC, Goldman JM, Cole PJ, Strickland B. Comparison of narrow section computed tomography and plain chest radiography in chronic allergic bronchopulmonary aspergillosis. Clin Radiol 1987; 38: 593-596. 